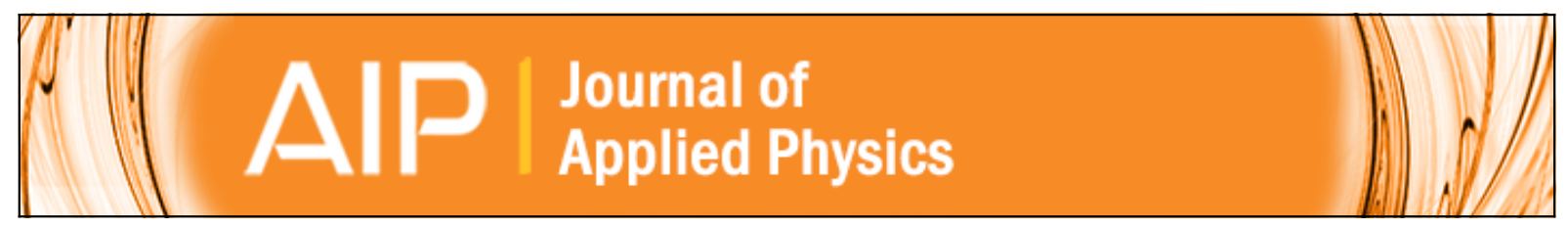

\title{
Domain wall release in "hard" piezoelectric under continuous large amplitude ac excitation
}

Yongkang Gao, Kenji Uchino, and Dwight Viehland

Citation: Journal of Applied Physics 101, 114110 (2007); doi: 10.1063/1.2745437

View online: http://dx.doi.org/10.1063/1.2745437

View Table of Contents: http://scitation.aip.org/content/aip/journal/jap/101/11?ver=pdfcov

Published by the AIP Publishing

\section{Articles you may be interested in}

Effective piezoelectric response of twin walls in ferroelectrics

J. Appl. Phys. 113, 187222 (2013); 10.1063/1.4801988

De-aging of Fe-doped lead-zirconate-titanate ceramics by electric field cycling: $180^{\circ}$ - vs. non- $180^{\circ}$ domain wall processes

J. Appl. Phys. 112, 034103 (2012); 10.1063/1.4739721

Evidence of temperature dependent domain wall dynamics in hard lead zirconate titanate piezoceramics J. Appl. Phys. 112, 014113 (2012); 10.1063/1.4736582

Evaluation of domain wall motion in bipolar fatigued lead-zirconate-titanate: A study on reversible and irreversible contributions

J. Appl. Phys. 107, 104119 (2010); 10.1063/1.3386461

Compression of piezoelectric ceramic at constant electric field: Energy absorption through non- $180^{\circ}$ domain-wall motion

J. Appl. Phys. 92, 1504 (2002); 10.1063/1.1489498

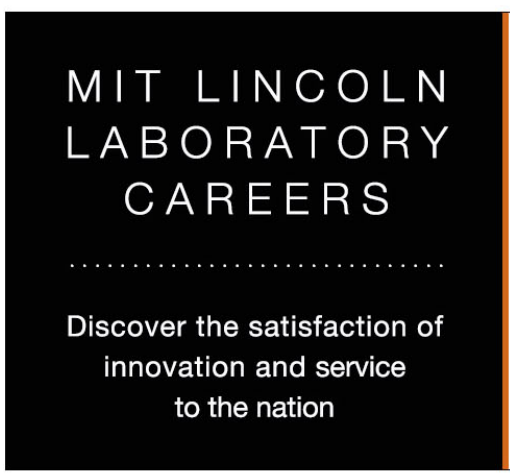

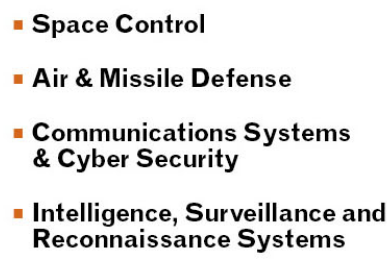

- Space Control

- Air \& Missile Defense

- Communications Systems \& Cyber Security

- Intelligence, Surveillance and

Reconnaissance Systems

- Advanced
Electronics
- Tactical Systems
- Homeland
Protection
- Air Traffic Control

LINCOLN LABORATORY

MAsSachusetts InStitute of TeChNOLOgY

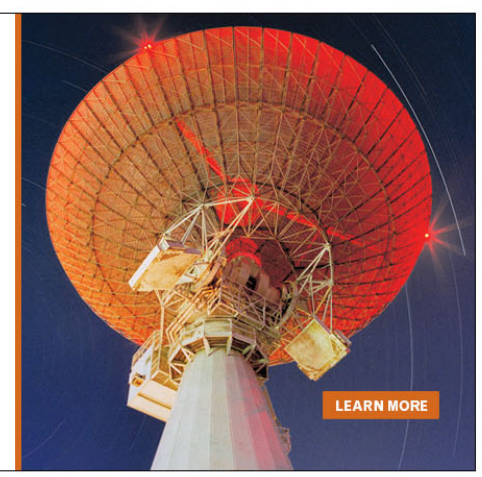




\title{
Domain wall release in "hard" piezoelectric under continuous large amplitude ac excitation
}

\author{
Yongkang $\mathrm{Gao}^{\mathrm{a})}$ \\ Hitchiner Manufacturing, Inc., 127 Old Wilton Road, Milford, New Hampshire 03055 \\ Kenji Uchino \\ International Center for Actuators and Transducers, Materials Research Laboratory, \\ Pennsylvania State University, University Park, Pennsylvania 16802 \\ Dwight Viehland \\ Department of Materials Science and Engineering, Virginia Tech, Blacksburg, Virginia 24060
}

(Received 10 September 2006; accepted 28 April 2007; published online 15 June 2007)

\begin{abstract}
Systematic investigation of large amplitude ac excitation effects on the polarization-electric-field $(P-E)$ and strain-electric-field $(\varepsilon-E)$ characteristics of both "hard" and "soft" $\mathrm{Pb}(\mathrm{Zr}, \mathrm{Ti}) \mathrm{O}_{3}$ ceramics was performed. Upon ac excitation, a gradual disappearance of the shift on $P-E$ response along the $E$ axis $\left(E_{\text {int }}\right)$ was observed for the hard piezoelectric; however, no such change was observed for the soft one. With increasing ac excitation cycle number, for a hard piezoelectric the internal dipolar field and mechanical quality factor $Q_{m}$ decrease by following an exponential time law, whereas the electromechanical and dielectric properties increase with significant deviation from the time law. It is proposed that additional domain release mechanism may exist for hard piezoelectric, besides the decrease of internal dipolar field upon continuous ac excitation. (C) 2007 American Institute of Physics. [DOI: 10.1063/1.2745437]
\end{abstract}

\section{INTRODUCTION}

High power piezoelectric materials offer increased energy density; thus, a reduction in the size of ultrasonic motors, piezoelectric transformers, and high power sonar can be achieved. For these electromechanical transduction applications, large ac electric fields need to be applied, which can be relatively high. The investigation of piezoelectric property change under continuous ac field excitation is a very important issue for both practical application and theoretical analysis of domain dynamics.

The internal dipolar field $\left(E_{\text {int }}\right)$ is shown as a shift of polarization-electric-field $(P-E)$ hysteresis loop along the $E$ axis (electric field) without applying external dc bias, which was found to be associated with acceptor dipole defect clusters. ${ }^{1-5}$ Aging of the physical properties in lower-valent iron-modified $\mathrm{Pb}(\mathrm{Zr}, \mathrm{Ti}) \mathrm{O}_{3}(\mathrm{PZT})$ was found to follow logarithmiclike responses in time domain and can be attributed to acceptor dipolar defects. ${ }^{5-7}$

Direct observation of a submicroscopic bending of domain walls between pinning sites was reported. ${ }^{8-10}$ Electron microscopy investigations of $K$-modified PZT have shown the development of "wavy" domains from normal micron sized domains when increasing $K$ concentration. Such wavy domains were attributed to the domain wall pinning effect.

In previous paper, ${ }^{11}$ we reported a significant increase of $Q_{m}$ with time $t$ for "hard" PZTs, whereas no such change was observed for the "soft" ones during the same time period. Correspondingly, a pronounced increase in $E_{\text {int }}$ was observed to follow $\ln (t)$. This observation could not be explained by traditional domain wall pinning model as $Q_{m}$ developed dur-

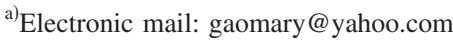

ing a relatively long time period (several hours after poling), which is very different from domain wall pinning process.

Application of continuous ac electric field on a fully aged Fe-modified PZT resulted in a gradual decrease in $E_{\text {int }}$ and corresponding gradual increase in the dielectric constant $K$. The "fresh" state was gradually recovered. ${ }^{12}$ So far, only few investigations were made on materials property changes during internal dipolar field relaxation process. Very few papers related the large signal parameter $(P-E$ hysteresis shift) to small signal materials property changes. In this paper, systematic investigation of $P-E$ hysteresis and related materials property changes upon continuous ac excitation will be conducted. A mechanism analysis of the piezoelectric property changes during this process will be performed. The experimental results suggested that besides internal dipolar field relaxation, additional domain wall release mechanism exists upon continuous large amplitude ac excitation in hard piezoelectric.

\section{EXPERIMENTAL PROCEDURE}

In this study the following compositions were investigated:

(1) $0.9 \mathrm{~Pb}\left(\mathrm{Zr}_{0.52} \mathrm{Ti}_{0.48}\right) \mathrm{O}_{3}-0.1 \mathrm{~Pb}\left(\mathrm{Sb}_{0.067} \mathrm{Mn}_{0.033}\right) \mathrm{O}_{3}-0.2$ at. $\%$ $\mathrm{RE}(\mathrm{Yb}, \mathrm{Nd}, \mathrm{La})$,

(2) commercialized hard PZT (APC 841, APC International Ltd.), and

(3) commercialized soft PZT (Type D, Taiheiyo Cement Corporation).

The samples were cut into $40 \times 7 \times 1 \mathrm{~mm}^{3}$ plates. The 

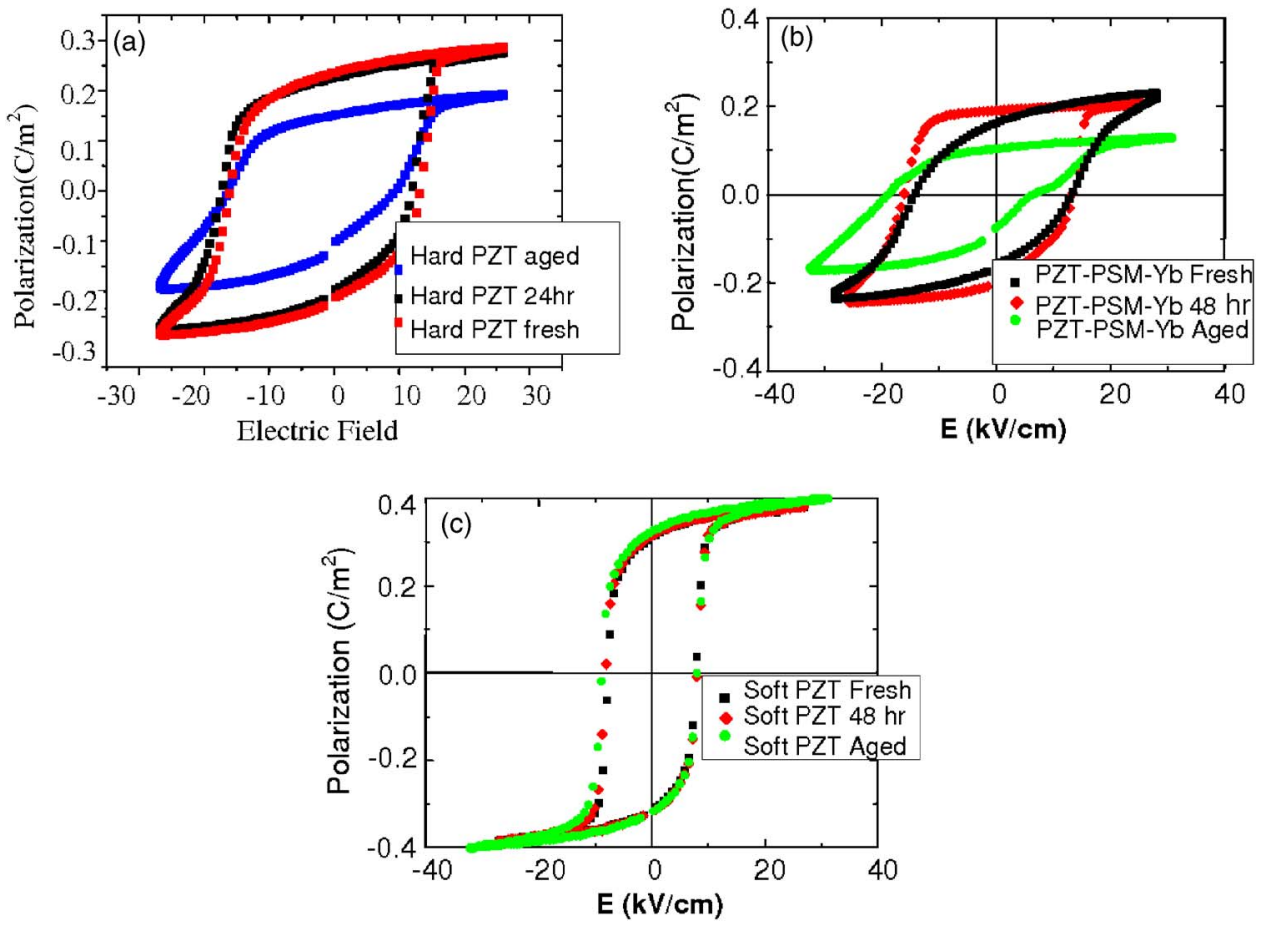

FIG. 1. (Color online) P-E response of (a) commercialized "hard" PZT, (b) RE doped PZT-PSM system, and (c) commercialized "soft" PZT during aging process (Ref. 11). poling process was conducted by immersing samples in silicon oil at $130{ }^{\circ} \mathrm{C}$ under an electric field of $30 \mathrm{kV} / \mathrm{cm}$ for $15 \mathrm{~min}$.

The electromechanical properties were measured by using a standard impedance analyzer. Spectra were obtained under a constant low-level voltage drive by using an HP4194 impedance analyzer. From the resonance $f_{r}$ (i.e., the admittance maximum) and the antiresonance $f_{a}$ (i.e., the admittance minimum) frequencies, the piezoelectric constant, the elastic compliance, the mechanical quality factor, and the electromechanical coupling coefficient were determined according to IEEE standard. ${ }^{13}$ For a rectangular piezoceramic plate, for example, the transverse coupling coefficient $k_{31}$ can be calculated as $k_{31}^{2} /\left(1-k_{31}^{2}\right)=\left(\pi^{2} / 4\right)\left(\Delta f / f_{r}\right)$, where $\Delta f=f_{a}$ $-f_{r}$. The mechanical quality factor $Q_{m}$ was calculated as $Q_{m}=f_{r} / f_{2}-f_{1}$, where $f_{r}$ is the resonance frequency and $f_{2} / f_{1}$ are the frequencies $3 \mathrm{~dB}$ down from the maximum admittance.

The $P-E$ and $\varepsilon-E$ curves (strain versus electric field) were measured by using a modified Sawyer-Tower circuit in conjunction with a linear variable differential transducer. The maximum amplitude of the applied ac electric field was as high as $30 \mathrm{kV} / \mathrm{cm}$. The frequency was $1 \mathrm{~Hz}$ (the time period for each cycle was $1 \mathrm{~s}$ ). For high field measurement, samples were immersed in the insulating liquid of Galden HT-200 to prevent arcing.

\section{EXPERIMENTAL RESULTS}

\section{A. $P-E$ and $\varepsilon-E$ characteristics}

Previously we observed the development of $E_{\text {int }}$ with time for hard piezoelectric ceramics. Figures 1(a)-1(c) show the $P-E$ response of commercialized hard PZT, rare earth modified $\mathrm{Pb}(\mathrm{Zr}, \mathrm{Ti}) \mathrm{O}_{3}-\mathrm{Pb}(\mathrm{Sb}, \mathrm{Mn}) \mathrm{O}_{3}$ (PZT-PSM-RE), and soft PZT during aging process, ${ }^{11}$ respectively. The measurements were conducted at $10 \mathrm{~min}, 48 \mathrm{~h}$, and one month after poling. At $10 \mathrm{~min}, P-E$ response was a symmetric loop for all of hard piezoelectric specimens, indicating that no significant $E_{\text {int }}$ was developed yet; however, after $48 \mathrm{~h}$, a slight shift of the $P-E$ response along the $E$ axis was found; whereas after one month, an $E_{\text {int }}$ field as large as $10 \mathrm{kV} / \mathrm{cm}$ was shown in the $P-E$ loop of PZT-PSM-RE. This is the highest $E_{\text {int }}$ observed among all of the investigated specimens. Contrary to hard piezoelectric, no such shift of $P-E$ response along the $E$ axis was observed for soft PZTs, as shown in Fig. 1(c), indicating that no $E_{\text {int }}$ was developed during the entire aging process.

In this paper, systematic investigation was conducted on the effect of continuous ac excitation on the $P-E$ and $\varepsilon-E$ responses of both hard and soft PZTs. Under continuous ac excitation, a relaxation of $E_{\text {int }}$ was found for hard-type piezoelectric ceramics, while no such change was observed for soft PZTs. Figure 2(a) shows the $P-E$ response of an aged commercialized hard PZT under continuous $30 \mathrm{kV} / \mathrm{cm}$ ac field. At the first cycle $N=1$, the $P-E$ response of the aged hard PZT showed a shift along the $E$ axis, with a remnant polarization $P_{r} \sim 0.15 \mathrm{C} / \mathrm{m}^{2}$; however, with increasing ac excitation cycle number $N, P-E$ loop gradually became symmetric and finally $E_{\text {int }}$ disappeared. At $N=360, P_{r}$ increased to $\sim 0.28 \mathrm{C} / \mathrm{m}^{2}$, which is close to but slightly higher than that of the "freshly" poled sample, as shown in Fig. 1(a).

Similar tendency was observed for PZT-PSM-RE. Figure 2(b) shows the $P-E$ relaxation process for PZT-PSM-RE. The aged specimen had an asymmetric $P-E$ loop and a significant shift along the $E$ axis; however, with increasing cycle number, the $P-E$ response gradually became symmetric. At $N=1, P_{r}$ was $\sim 0.18 \mathrm{C} / \mathrm{m}^{2}$; at $N=360, P_{r}$ increased to $\sim 0.35 \mathrm{C} / \mathrm{m}^{2}$. This result indicates that with increasing ac excitation cycle number, the domain stabilizing force decreases and the domain becomes switchable again. 

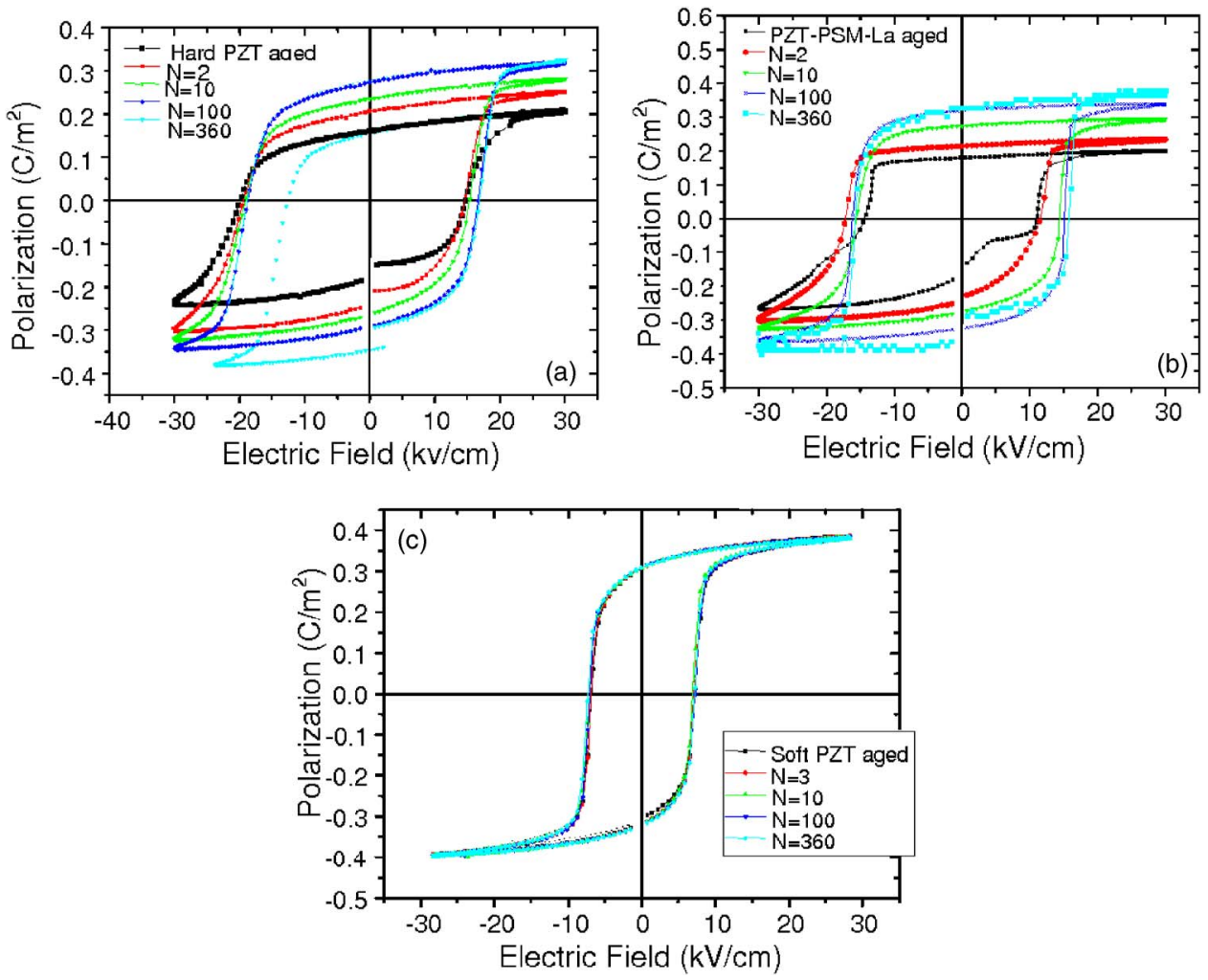

FIG. 2. (Color online) $P-E$ hysteresis relaxation of (a) commercialized "hard" PZT, (b) RE doped PZT-PSM system, and (c) commercialized "soft" PZT.
Contrary to hard-type PZTs, soft PZT did not show P-E relaxation under continuous ac excitation. No such change of $P-E$ response was observed during ac excitation process, as shown in Fig. 2(c). It is clear that with or without $E_{\text {int }}$ is the root difference between hard and soft PZTs.

Figure 3 summarizes $P_{r}$ versus cycle number $N$ for both commercialized hard PZT and PZT-PSM-RE. For both materials, $P_{r}$ increased with increasing $N$. Continuous ac excitation resulted in the decrease of domain stabilizing force $E_{\text {int }}$ or domain wall releasing from pinning sites, which may contribute to the increase of switchable polarization.

The value of $E_{\text {int }}$ can be determined by differential changes of the $P-E$ response between consecutive measurements. Naturally, this method has the limitation that noise is introduced by numerical differentiation. Figure 4 shows the

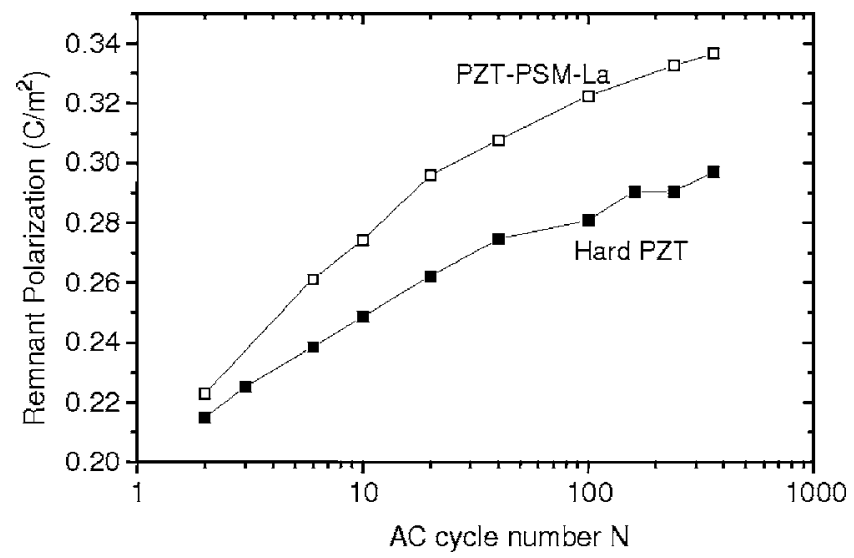

FIG. 3. The remanent polarization $P_{r}$ increase as a function of ac cycle number $N$ for commercialized "hard" PZT and RE doped PZT-PSM ceramics. calculated $E_{\text {int }}$ as a function of $N$. If we define time $t$ as the product of time period for each cycle ( $1 \mathrm{~s}$ for this study) and cycle number $N$, the decrease of $E_{\text {int }}$ with time can be well fitted to the exponential relation

$$
E_{\text {int }}(t)=E_{\text {int }}(0) \exp (-t / \tau),
$$

where $E_{\text {int }}(0)$ is the internal dipolar field in the aged condition and $\tau$ is a characteristic relaxation time. We found this equation valid only within limited ranges of temperature $T$ and field strength $E$, as $\tau$ depends on both $T$ and $E$.

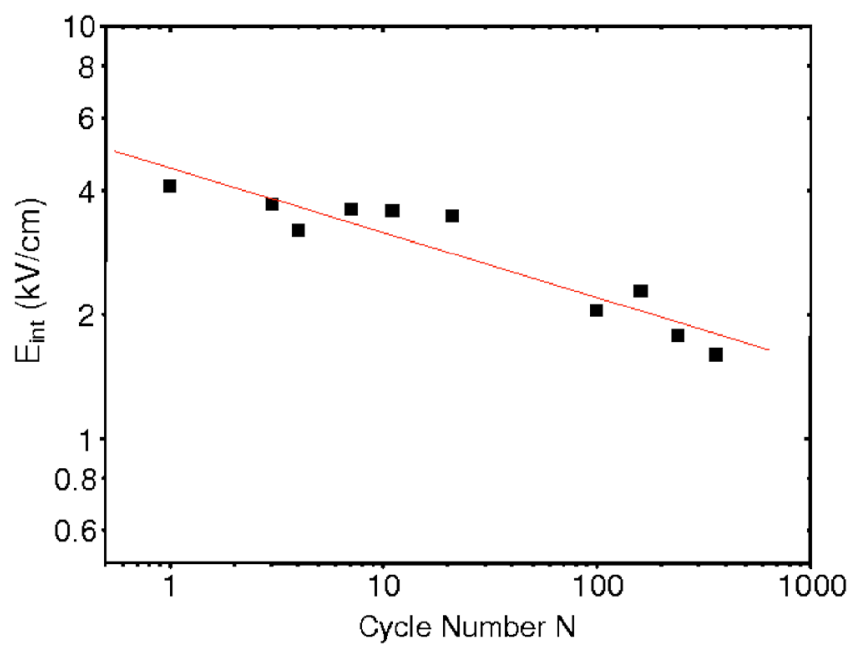

FIG. 4. (Color online) Calculated internal dipolar field as a function of ac cycle number $N$ for commercial "hard" PZTs. 


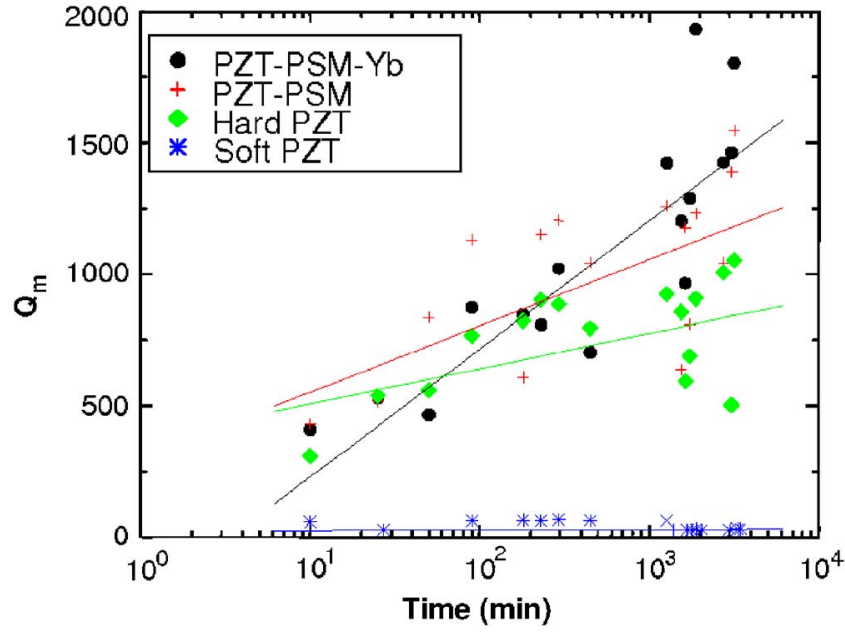

FIG. 5. (Color online) $Q_{m}$ vs time for commercialized "hard" PZTs, PZTPSM-RE, and "soft" PZTs within $48 \mathrm{~h}$ (2880 min) after poling (Ref. 11).

\section{B. Resultant property changes due to relaxation of $E_{\text {int }}$}

Previously we observed a significant increase of the mechanical quality factor $Q_{m}$ as a function of time after poling. ${ }^{11}$ Figure 5 shows the $Q_{m}$ versus time for commercialized hard PZTs, PZT-PSM-RE, and soft PZTs within $48 \mathrm{~h}$ (2880 min) after poling. The initial $Q_{m}$ value of PZTPSM-RE was only 400 at 10 min after poling; however, at 2880 min after poling, $Q_{m}$ increased up to 1600 . The $Q_{m}$ value increased significantly for hard piezoelectrics and the increasing rate seems to be related to the degree of "hardness:" the harder the materials are, the higher the $Q_{m}$ increasing rate is. However, for soft PZT no $Q_{m}$ change was observed within the same time frame. The pronounced increase of both $E_{\text {int }}$ and $Q_{m}$ was observed to follow a logarithm time relationship $\ln (t)$, which is relatively slow and significantly different from exponential relationship as $E_{\text {int }}$ relaxes during ac excitation process, as shown in Fig. 4 and Eq. (1). The development of $E_{\text {int }}$ occurs very gradually because oxygen vacancy diffusion takes relatively long time. ${ }^{11}$ The developed internal dipolar fields clamp polarization switching, via a conjugate coupling to $P$. The slow development of $Q_{m}$ and $E_{\text {int }}$ at room temperature is contradictory to the traditional domain wall pinning model as at room temperature pinning sites should not have enough mobility to diffuse to domain boundaries, when $Q_{m}$ and $E_{\text {int }}$ develop.

The deaging process caused by ac excitation is much faster than aging process. The oxygen diffusion rate is very slow compared to external ac cycle rate. The oxygen vacancy-acceptor defect dipoles do not have enough time to reorient to the direction parallel to spontaneous polarization during each ac cycle; therefore $E_{\text {int }}$ decreased at a much higher rate upon ac excitation as compared to the process when $E_{\text {int }}$ develops.

Internal dipolar fields $E_{\text {int }}$ play an important role in domain stabilization. The reduced $E_{\text {int }}$ will result in an enhancement in the physical properties and a decrease in the mechanical quality factor.

Figures 6(a)-6(c) show how electromechanical coupling coefficient $k_{31}$ changes during internal dipolar field relaxation process for a commercialized hard PZT, PZT-PSM-RE, and commercialized soft PZT, respectively. With increasing time $t$ (or ac cycle number $N$ ), $k_{31}$ can be seen to increase for both commercialized hard PZT and PZT-PSM-RE. The $k_{31}$ dependence on $t$ (or $N$ ) deviated from the exponential time law as shown in Eq. (1), which $E_{\text {int }}$ follows during the relaxation process. This result suggests that additional domain
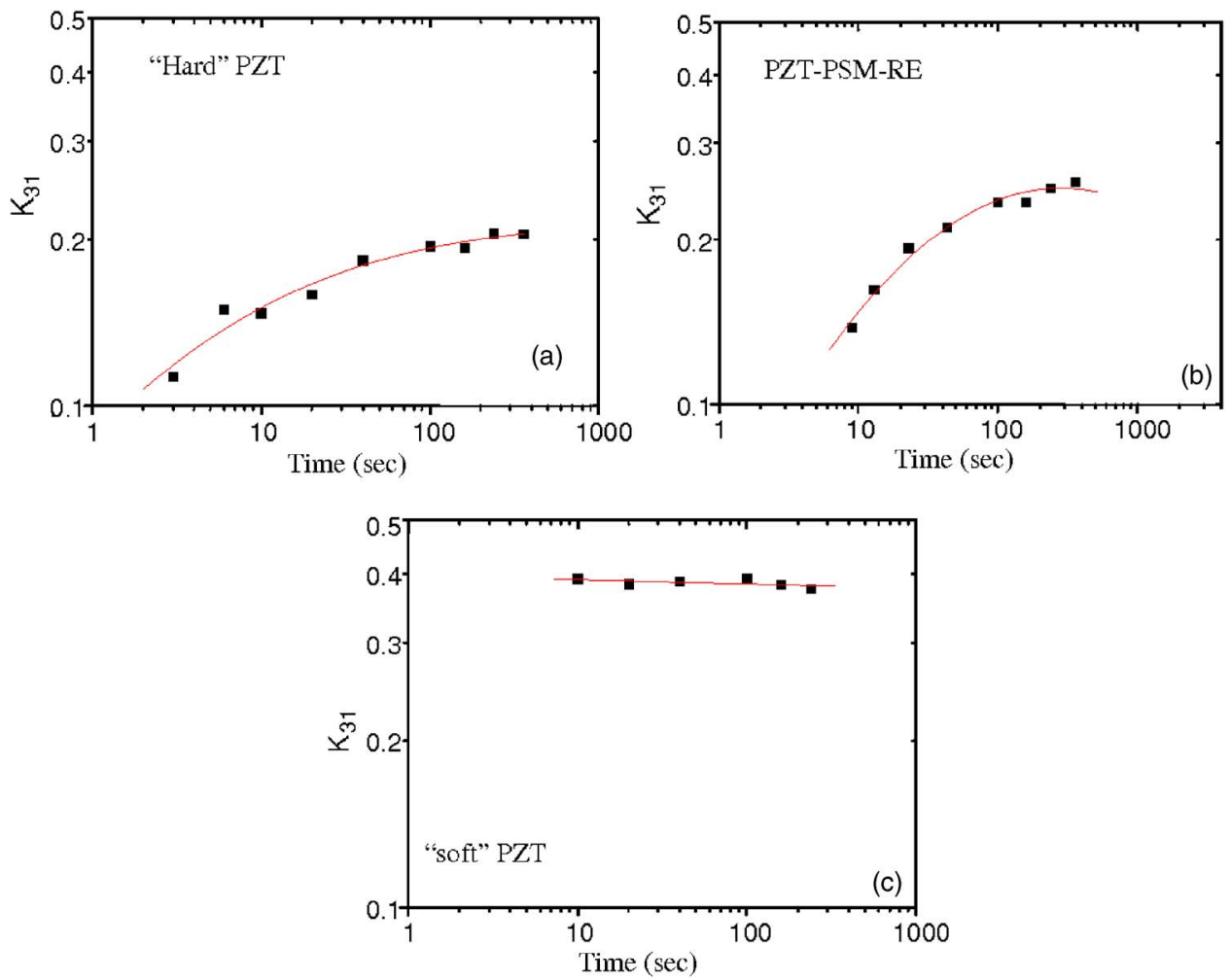

FIG. 6. (Color online) Electromechanical coupling coefficient $k_{31}$ value change during internal dipolar field relaxation process for (a) commercialized "hard" PZT, (b) RE doped PZTPSM, and (c) commercialized "soft" PZT. 


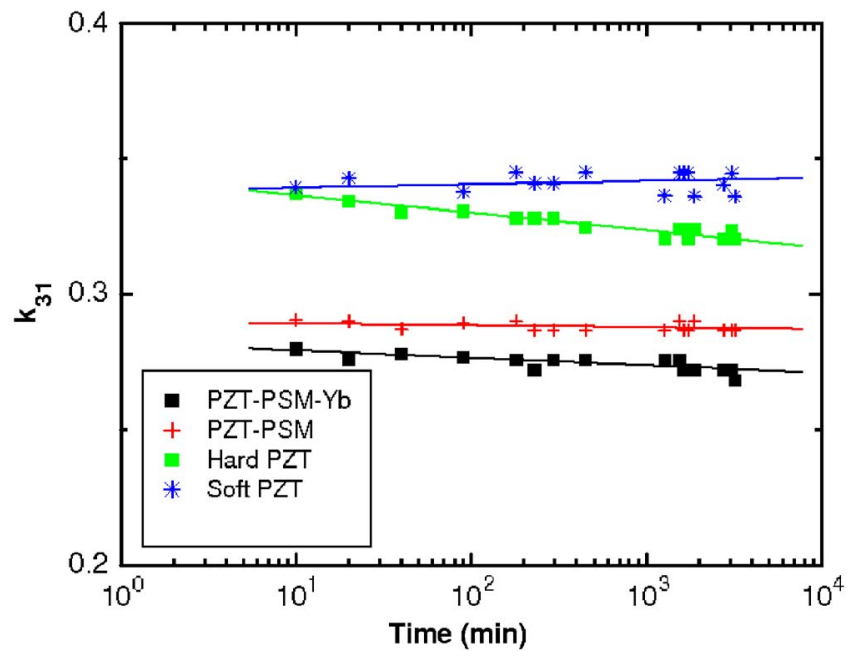

FIG. 7. (Color online) Electromechanical coupling coefficient $k_{31}$ value change during aging with the development of internal dipolar field $E_{\text {int }}$ for commercialized "hard" PZT, RE doped PZT-PSM, and commercialized "soft" PZT.

release mechanism besides the decrease of $E_{\text {int }}$ may contribute to the electromechanical performance of hard piezoelectric upon continuous ac excitation. In addition, it is important to notice that the increase of $k_{31}$ was quite significant for hard piezoelectric ceramics upon ac excitation, whereas no such change was observed for soft PZTs. At $t=360 \mathrm{~s}(N$ $=360), k_{31}$ increased by 0.12 for PZT-PSM-RE; however, $k_{31}$ decreased only by 0.01 during the aging process, as shown in Fig. $7 .^{11}$ It is very clear that there is a significant difference of electromechanical property changes between aging and deaging processes. Besides the decrease of $E_{\text {int }}$, additional domain release mechanism may exist which contribute to the significant $k_{31}$ increase during ac excitation process. Uchida-

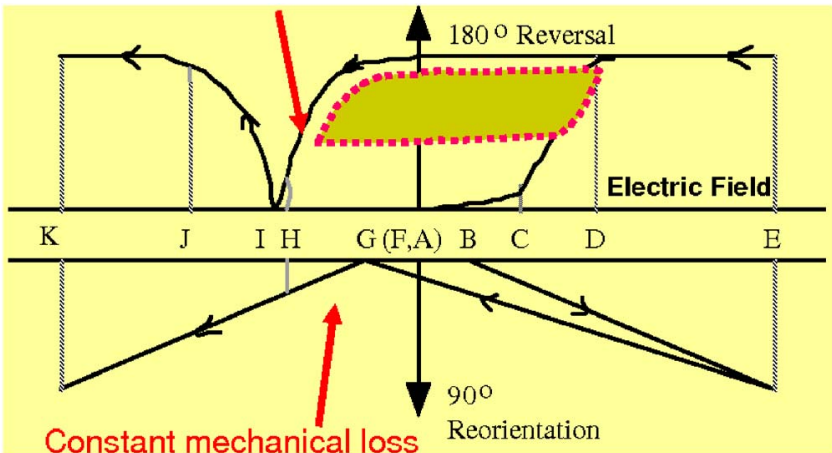

FIG. 8. (Color online) Uchida-Ichida model (Refs. 14 and 15) was applied to understand how $E_{\text {int }}$ affects $Q_{m}$ and other electromechanical properties of "hard" piezoelectric.

Ichida model ${ }^{14,15}$ was applied to understand how $E_{\text {int }}$ affects $Q_{m}$ and other electromechanical properties of hard piezoelectric, as shown in Fig. 8. $E_{\text {int }}$ has a similar effect as external positive dc bias on domain dynamics. Electrical field has only gradual influence on $90^{\circ}$ domain switching, whereas above certain field level, it could significantly affect $180^{\circ}$ domain reversal. ${ }^{15}$ It was believed that mechanical loss is mainly associated with $90^{\circ}$ domain switching whereas dielectric loss is associated with $180^{\circ}$ domain reversal. ${ }^{16}$ At resonance heat generation is dominantly contributed by intensive mechanical loss. Considering that intensive mechanical loss consists of extensive dielectric loss; ${ }^{16}$ above a certain field level extensive dielectric loss increases drastically (as shown in Fig. 8); thus the mechanical quality factor $Q_{m}$ (inverse value of mechanical loss) should be a field sensitive parameter, whereas $k_{31}$ should not be very sensitive to electric field. The effects of $E_{\text {int }}$ on $Q_{m}$ and other electromechanical properties during aging process after poling supported the
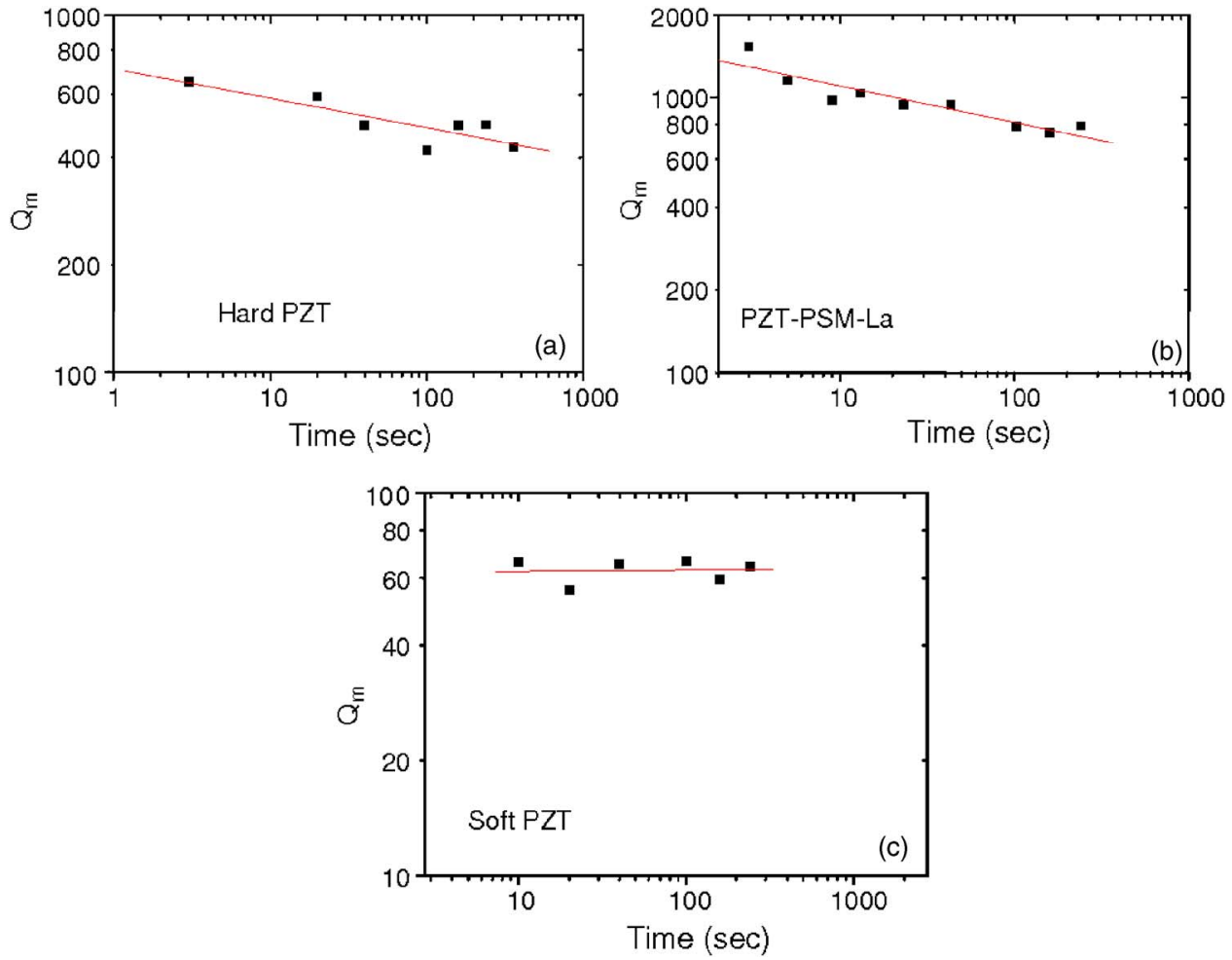

FIG. 9. (Color online) Mechanical quality factor $Q_{m}$ value change during internal dipolar field relaxation process for (a) commercialized "hard" PZT, (b) RE doped PZT-PSM, and (c) commercialized "soft" PZT. 

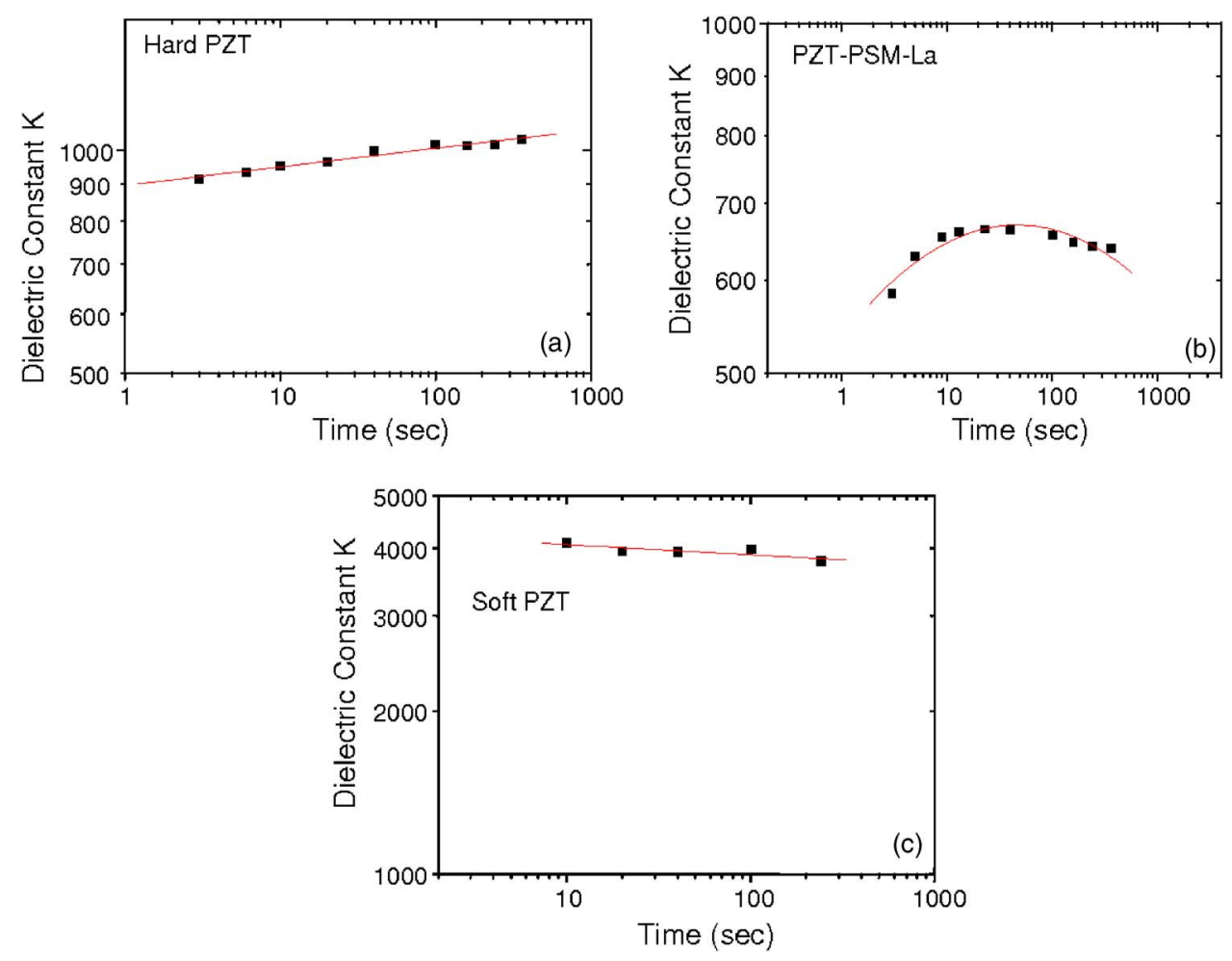

FIG. 10. (Color online) Dielectric constant $K$ value change during internal dipolar field relaxation process for (a) commercialized "hard" PZT, (b) RE doped PZT-PSM, and (c) commercialized "soft" PZT.

conjecture that $E_{\text {int }}$ has a significant impact on $Q_{m}$ but not on $k_{31}{ }^{11}$ The possible explanation for the significant $k_{31}$ increase upon large amplitude ac excitation could be attributed to the depinning of defect clusters from domain boundaries, in addition to $E_{\text {int }}$ decrease. Internal dipolar field could be formed by reorienting the dipolar defects via oxygen diffusion and the defect clusters do not necessarily need to be accumulated around the domain boundaries. However, to form domain wall pinning, defect complexes need to physically diffuse into domain boundaries and pin the walls. Internal dipolar field and domain wall pinning effects could be somewhere overlapping but different mechanisms contribute to domain dynamics.

Figures 9(a)-9(c) show $Q_{m}$ as a function of time for commercialized hard PZT, PZT-PSM-RE, and commercialized soft PZT upon continuous ac excitation, respectively. The $Q_{m}$ can be seen to decrease with time for hard piezoelectric ceramics [Figs. 9(a) and 9(b)] by following an exponential relation $\exp (-t / \tau)$ as shown in Eq. (1), whereas no such change was observed for soft PZT [Fig. 9(c)]. This observation is consistent with the conjecture that $E_{\text {int }}$ has a large impact on $Q_{m}$ but not on electromechanical properties. The pronounced decrease of mechanical quality factor for hard piezoelectric ceramics during ac excitation accompanies the increased energy transduction from electrical to mechanical forms, as $k_{31}$ increases. More mechanical energy is transformed from electrical energy, but a higher fraction of energy is dissipated as heat. Electrically induced shape changes which occur on domain release are inherently dissipative. The changes in $Q_{m}$ have a large impact on the application of high power transducer materials, as mechanical loss factor contributes dominantly to the heat generation under resonance drive. The decrease of $Q_{m}$ and $E_{\text {int }}$ during ac excitation limits possible driving life of high power operations.
Figures $10(\mathrm{a})-10(\mathrm{c})$ show the dielectric constant $K$ as a function of time for a commercialized hard PZT, PZT-PSM$\mathrm{RE}$, and commercialized soft PZT, respectively. $K$ was observed to increase with time upon ac excitation for "hard" piezoelectric [Figs. 10(a) and 10(b)] however, a slight decrease of $K$ was observed for soft PZT [Fig. 10(c)], which may be caused by microcrack generated during large amplitude ac excitation. A significant deviation from exponential time relationship was observed for PZT-PSM-RE specimens. $K$ increased first and then decreased with further increasing ac excitation cycle number. PZT-PSM modified with various rare earth elements were investigated; however, similar tendency was observed for each specimen. The mechanism is not clear yet. Contrary to hard PZTs, no increase of dielectric constant, electromechanical properties, and decrease of $Q_{m}$ were observed for soft PZT upon continuous ac excitation.

\section{SUMMARY}

Systematic investigation of $P-E, \varepsilon-E$, and related electromechanical property change of both "hard" and "soft" piezoelectrics upon continuous large amplitude ac excitation was performed. $E_{\text {int }}$ decreased with increasing ac cycle number, by following an exponential time relationship $\exp (-t / \tau)$. The decrease of $E_{\text {int }}$ during ac excitation much faster than $E_{\text {int }}$ develops by following $\ln (t)$ during aging process. The slow increase of $Q_{m}$ and $E_{\text {int }}$ during aging is contradictory to the traditional domain wall pinning model. The relaxation of $E_{\text {int }}$ was shown to result in an increased transient coupling coefficient $k_{31}$. However, the increase of energy transduction from electrical to mechanical forms is accompanied by significantly increased mechanical energy dissipation as heat. For hard piezoelectric, $k_{31}$ increased significantly upon continuous ac excitation, with a large devia- 
tion from the exponential time law, whereas $E_{\text {int }}$ follows $\exp (-t / \tau)$ during relaxation. This observation indicates that additional domain release mechanism which contributes to enhanced electromechanical response exists besides the decrease of $E_{\text {int }}$ upon ac excitation.

${ }^{1}$ H. H. Wieder, J. Appl. Phys. 30, 1010 (1959).

${ }^{2}$ U. Robels, J. H. Calderwood, and G. Arlt, J. Appl. Phys. 77, 4002 (1995).

${ }^{3}$ E. T. Keve, K. L. Bye, P. W. Whipps, and A. S. Annis, Ferroelectrics 3, 39 (1971).

${ }^{4}$ K. Carl and K. H. Hardtl, Ferroelectrics 17, 473 (1978).

${ }^{5}$ U. Robels and G. Arlt, J. Appl. Phys. 73, 3454 (1993).

${ }^{6}$ U. Robels, R. Lohkamper, and G. Arlt, Proceedings of the ISAF'90, Urbana, IL, 1990 (unpublished).
${ }^{7}$ U. Robels, R. Lohkamper, and G. Arlt, IEEE Ultrasonic Symposium, 1991 (unpublished), p. 44.

${ }^{8}$ T. J. Yang, V. Gopalan, P. J. Swart, and U. Mohideen, Phys. Rev. Lett. 82, 4106 (1999).

${ }^{9}$ Q. Tan, J. F. Li, and D. Viehland, Philos. Mag. B 76, 59 (1997).

${ }^{10}$ Q. Tan, J. Li, and D. Viehland, Appl. Phys. Lett. 75, 418 (1999).

${ }^{11}$ Y. Gao, K. Uchino, and D. Viehland, Jpn. J. Appl. Phys., Part 1 45, 12 (1996).

${ }^{12}$ K. Carl and K. H. Hardt, Ferroelectrics 17, 473 (1978).

${ }^{13}$ IRE Standard (1957).

${ }^{14}$ Y. Gao, K. Uchino, and D. Viehland, J. Appl. Phys. 101, 054109 (2007).

${ }^{15}$ N. Uchida and T. Ikeda, Jpn. J. Appl. Phys. 4, 867 (1965).

${ }^{16}$ K. Uchino, IEEE Trans. Ultrason. Ferroelectr. Freq. Control 48, 307 (2001). 